\title{
Prediction of protein-protein interactions between viruses and human by an SVM model
}

\author{
Guangyu Cui, Chao Fang, Kyungsook Han \\ From The 2011 International Conference on Intelligent Computing (ICIC 2011) \\ Zhengzhou, China. 11-14 August 2011
}

\begin{abstract}
Background: Several computational methods have been developed to predict protein-protein interactions from amino acid sequences, but most of those methods are intended for the interactions within a species rather than for interactions across different species. Methods for predicting interactions between homogeneous proteins are not appropriate for finding those between heterogeneous proteins since they do not distinguish the interactions between proteins of the same species from those of different species.

Results: We developed a new method for representing a protein sequence of variable length in a frequency vector of fixed length, which encodes the relative frequency of three consecutive amino acids of a sequence. We built a support vector machine (SVM) model to predict human proteins that interact with virus proteins. In two types of viruses, human papillomaviruses (HPV) and hepatitis $C$ virus (HCV), our SVM model achieved an average accuracy above $80 \%$, which is higher than that of another SVM model with a different representation scheme. Using the SVM model and Gene Ontology (GO) annotations of proteins, we predicted new interactions between virus proteins and human proteins.
\end{abstract}

Conclusions: Encoding the relative frequency of amino acid triplets of a protein sequence is a simple yet powerful representation method for predicting protein-protein interactions across different species. The representation method has several advantages: (1) it enables a prediction model to achieve a better performance than other representations, (2) it generates feature vectors of fixed length regardless of the sequence length, and (3) the same representation is applicable to different types of proteins.

\section{Background}

A variety of viruses cause diseases in humans, and viral infections affect millions of people each year. The treatment and prevention of viral infections and their associated diseases are the main public health challenges. Common examples of viruses associated with human diseases include HIV-1, influenza virus, human papillomavirus (HPV), herpes virus, and hepatitis A, B, C, D and E viruses. Different viral species have different infection mechanisms, and identifying host cell proteins that are attacked by a virus will certainly help better understand the infection mechanism and to design new antiviral strategies. Recently, proteome-wide studies of viral interactions with human

\footnotetext{
* Correspondence: khan@inha.ac.kr

School of Computer Science and Engineering, Inha University, Incheon, South Korea
}

proteins were performed, but comprehensive analysis of the interactions between virus proteins and human proteins involved in viral infection has not yet been investigated.

So far, most computational studies of protein-protein interactions (PPIs) have focused on the interactions between proteins of the same species. For example, Bock and Gough [1] created protein structural and physiochemical descriptors based on the sequence data, and trained a support vector machine classifier to identify PPIs from the descriptors. There are many other studies that used a support vector machine to predict PPIs in several organisms [2-6].Wu et al. [7] used the Gene Ontology (GO) and other annotations to predict PPIs in yeast. Nanni [8] predicted PPIs in the human gastric bacterium Helicobacter pylori by combining a linear discriminant classifier and 
cloud points. You et al. [9] used a manifold embedding method to assess and predict PPIs. All these methods are intended for the protein-protein interactions within a species rather than for those across different species. Methods typically used to predict interactions between homogeneous proteins are not appropriate for predicting those between heterogeneous proteins, since such methods do not distinguish interactions between proteins of the same species from those of different species.

In this paper, we propose a representation method and a support vector machine (SVM) model to predict the interactions between two types of viruses (hepatitis $C$ virus and human papillomaviruses) and human proteins. Although substantial progress has been made in clinical and basic research on the hepatitis $\mathrm{C}$ virus $(\mathrm{HCV})$ and human papillomaviruses (HPV), interactions between these viruses and human proteins are not yet fully understood. Identifying more interactions between them should help elucidate the interaction mechanism of HCV and HPV with host cells, and can be helpful in designing molecules that target the new interacting proteins.

\section{Methods}

\section{Representation of protein sequences}

One of the challenges in predicting protein-protein interactions from sequences is to find a way of fully encoding the important information content of protein sequences. In addition, the amino acid sequences of different lengths should be transformed into a feature vector of the same length. We represent a protein sequence using three consecutive amino acids called amino acid triplet. For example, in the amino acid sequence TVAVTVA, there are four overlapping amino acid triplets, TVA, VAV, AVT and VTV. To reduce the dimension of the vector space of feature vectors, we represent an amino acid sequence using the class of amino acids. Based on the biochemical similarity of amino acids, twenty amino acids were classified into six categories: $\{$ IVLM $\},\{F Y W\},\{H K R\},\{D E\}$, $\{$ QNTP $\}$, and $\{$ ACGS $\}[10,11]$. Using this classification of amino acids, there are $6 \times 6 \times 6=216$ possible amino acid triplets.

We use a binary space $(\mathrm{V}, \mathrm{F})$ to represent a protein sequence, in which $\mathrm{V}$ is a vector space of feature vectors with a fixed number of features and $F$ is a vector space of frequency vectors. A protein sequence of variable length is first mapped to a feature vector of fixed length. A feature vector $v$ is then mapped to a relative frequency vector $d_{i}$ $(i=1,2, \ldots, 216)$, which is defined by equation 1 .

$$
d_{i}=\left\{e^{\frac{f i-\min \left\{f_{1}, f_{2}, \ldots, f_{216}\right\}}{\max \left\{f_{1}, f_{2}, \ldots, f_{216}\right\}-\min \left\{f_{1}, f_{2}, \ldots, f_{216}\right\}}}\right\}-1
$$

where $f_{i}$ is the frequency of the $i$-th triplet type in the sequence. The value of $d_{i}$ ranges from 0 to 1.714 .

There are two differences between our representation and that of Shen et al. [5], namely in the classification of amino acids and in the definition of the relative frequency of an amino acid triplet. First, Shen et al. [5] clustered the 20 amino acids into seven classes, $\{A V G\}$, $\{\mathrm{ILFP}\},\{\mathrm{YMTS}\},\{\mathrm{HNQW}\},\{\mathrm{RK}\},\{\mathrm{DE}\},\{\mathrm{C}\}$, based on the diploes and volumes of the side chains of amino acids, and there are $7 \times 7 \times 7=343$ possible amino acid triplets. Second, the relative frequency of a triplet in their representation is defined by equation 2 .

$$
d_{i}=\frac{f_{i}-\min \left\{f_{1}, f_{2}, \ldots, f_{343}\right\}}{\max \left\{f_{1}, f_{2}, \ldots, f_{343}\right\}-\min \left\{f_{1}, f_{2}, \ldots, f_{343}\right\}}
$$

While the relative frequency in the representation of Shen et al. [5] has a value in the range 0[1], it ranges from 0 to 1.714 in our representation. Thus, the relative frequency value in a wider range makes it easier to discriminate protein sequences as we will show later in the results section.

In addition to the relative frequencies of amino acid triplets, a feature element representing the types of virus proteins (11 types of HCV proteins and 9 types of HPV proteins) were included in a feature vector. Each feature vector was labelled +1 for interaction and -1 for noninteraction. By encoding the type of a virus protein, the SVM model can find a human protein interacting with the virus protein.

\section{Support vector machine}

A support vector machine (SVM) has been applied to several biological problems such as prediction of protein-protein interactions [1-6], homology detection [12], and analysis of gene expression data [13]. Data examples labelled positive or negative are projected into a highdimensional feature space using a kernel, and the hyperplane in the feature space is optimized to maximize the margin between positive and negative data examples. We implemented an SVM model using LIBSVM http:// www.csie.ntu.edu.tw/ cjlin/libsvm/ with the radial basis function (RBF) as a kernel function, which is defined by

$$
K(u, v)=\exp \left(-\gamma\|u-v\|^{2}\right)
$$

where $u$ and $v$ are two data vectors and $\gamma$ is a training parameter. A smaller $\gamma$ value makes the decision boundary smoother. The regularization factor $\mathrm{C}$ controls trade-off between a low training error and a large margin [14]. In this study, we set $C=20$ and $\gamma=0.1$.

We tried several other kernel functions with our data. The linear and polynomial kernel functions resulted in high sensitivity (almost 100\%), but low specificity (about 
$50 \%)$. The sigmoid kernel function showed poor performance both in sensitivity and specificity (about 50\%). The radial basis function was the only one that showed reasonably good sensitivity and specificity, and chosen as the kernel function of the SVM model.

\section{Data set of viral interaction with human proteins}

Hepatitis $\mathrm{C}$ virus $(\mathrm{HCV})$ is a small enveloped virus with a single-stranded RNA genome encoding a single open reading frame [15]. The polyprotein of approximately 3,100 amino acids is cleaved into the structural proteins (core, E1 and E2), hydrophobic peptide p7, and nonstructural proteins such as NS2, NS3, NS4A, NS4B, NS5A and NS5B [16]. Although many experimental studies have been performed so far, the underlying mechanisms controlling the entry of $\mathrm{HCV}$ into host cells and interactions with the host cells are not fully known, and an efficient treatment for $\mathrm{HCV}$ infection has not yet been developed.

We obtained the interaction data between HCV proteins and human proteins from the infection mapping project (I-MAP) [17]. I-MAP provides a comprehensive view of viral infections at the protein level by mapping the interactions of a large amount of viral proteins with host proteins. There are 481 interactions between 11 HCV proteins and 421 human proteins. By searching Gene IDs of the 421 human proteins in HPRD http:// www.hprd.org, we identified a total of 695 interactions between HCV proteins and human proteins. The 695 protein-protein interactions formed a positive data set for an SVM model. Both positive and negative data are required to train an SVM model. Unlike positive data, negative samples are not readily available from proteinprotein interaction data. We randomly selected 695 human proteins from HPRD, which were not included in the positive data set, and generate a negative data set with them. Our method of generating a negative data set is similar to that of Gomez et al. [10], which assumes a negative protein interaction if there is no explicit evidence of an interaction. Since an unbalance between positive and negative data sets introduces a prediction bias, we generated a negative data set with the same number of proteins as the positive data set.

For evaluating an SVM model, we divided both the positive and negative data sets into training and test sets. We randomly selected 500 positive data and 500 negative data for a training set. The remaining 195 positive data and 195 negative data were used to construct a test set. To keep the same proportion of human proteins interacting with each virus protein in both training and test sets, we selected training data by

$$
N_{i}=N(\text { Training }) \cdot \frac{N\left(T_{i}\right)}{N(\text { Total })}
$$

where $T_{i}$ is the $i$-th virus protein $(i=1,2, \ldots, 11$ in $\mathrm{HCV}), \mathrm{N}\left(T_{i}\right)$ is the number of human proteins interacting with the $i$-th $\mathrm{HCV}$ protein, $\mathrm{N}$ (Training) is the total number of positive training data, and $\mathrm{N}$ (Total) is the total number of HCV-human protein interactions.

Table 1 shows the numbers of human proteins known to interact with each $\mathrm{HCV}$ protein, and those selected for a training set. For example, 298 human proteins are known to interact with the HCV NS3 protein. 214 out of the 298 human proteins were randomly selected as positive data of a training set since $\mathrm{N}(\mathrm{NS} 3)=500 \times 298$ / $695=214$. We selected the same number of human proteins from a negative dataset as negative interaction partners of the HCV NS3 protein.

Human papillomavirus (HPV) is a member of the papillomavirus family of viruses that is capable of infecting humans. HPV types 16 and 18 cause $70 \%$ of cervical cancer $[18,19]$. So far, a small number of host proteins have been known to interact with HPV proteins, so a systematic prediction of large-scale interactions between HPV proteins and human proteins would help find new human proteins targeted by HPV. We extracted the interactions of HPV-16 and HPV-18 proteins with human proteins from the NCBI BioSystems Database (http://www.ncbi.nlm.nih.gov/biosystems/). After removing redundancy, we identified a total of 252 interactions of HPV proteins with human proteins, and obtained Gene IDs from HPRD http://www.hprd.org. A training set and test set for HPV interactions were constructed in the same way as for the HCV interactions. Table 2 shows the numbers of human proteins known to interact with

\begin{tabular}{|c|c|c|}
\hline HCV protein & Number of $\mathrm{H}_{\mathrm{HCV}}$ & Number of $\mathrm{H}_{\mathrm{HCV}}$ in a training set \\
\hline core & 118 & 85 \\
\hline E1 & 16 & 12 \\
\hline E2 & 29 & 21 \\
\hline $\mathrm{F}$ & 10 & 7 \\
\hline NS2 & 11 & 8 \\
\hline NS3 & 298 & 214 \\
\hline NS4A & 7 & 5 \\
\hline NS4B & 1 & 1 \\
\hline NS5A & 152 & 109 \\
\hline NS5B & 36 & 26 \\
\hline p7 & 17 & 12 \\
\hline Total & 695 & 500 \\
\hline
\end{tabular}

$\mathrm{H}_{\mathrm{HCV}}$ represents the human proteins that are known to interact with $\mathrm{HCV}$ proteins. For each $\mathrm{HCV}$ protein, the number of $\mathrm{H}_{\mathrm{HCV}}$ in a training set was computed by $N_{i}=N($ Training $) \cdot \frac{N\left(T_{i}\right)}{N(\text { Total })}$, where $T_{i}$ is the $i$-th HCV protein. 
Table 2 The number of human proteins interacting with HPV proteins

\begin{tabular}{ccc}
\hline HPV protein & Number of $\mathbf{H}_{\mathbf{H P V}}$ & Number of $\mathbf{H}_{\mathbf{H P V}}$ in a training set \\
\hline E1 & 9 & 7 \\
E2 & 36 & 29 \\
E4 & 2 & 2 \\
E5 & 13 & 10 \\
E6 & 78 & 62 \\
E7 & 76 & 60 \\
E8 & 7 & 6 \\
L1 & 20 & 16 \\
L2 & 11 & 8 \\
\hline Total & 252 & 200
\end{tabular}

$\mathrm{H}_{\mathrm{HPV}}$ represents the human proteins that are known to interact with HPV proteins. For each HPV protein, the number of $\mathrm{H}_{\mathrm{HPV}}$ in a training set was computed by $N_{i}=N($ Training $) \cdot \frac{N\left(T_{i}\right)}{N(\text { Total })}$, where $\mathrm{T}_{\mathrm{i}}$ is the $\mathrm{i}$-th HPV protein.

each HPV protein and those used to train an SVM model for HPV interactions.

\section{Results and discussion}

\section{Performance evaluation}

We evaluated the performance of the SVM model in terms of sensitivity, specificity and accuracy.

$$
\begin{aligned}
& \text { Sensitivity }=\frac{T P}{T P+F N} \\
& \text { Specificity }=\frac{T N}{T N+F P} \\
& \text { Accuracy }=\frac{T P+T N}{T P+F P+T N+F N}
\end{aligned}
$$

True positives (TP) are actual interacting proteins that are predicted correctly. True negatives (TN) are noninteracting proteins that are predicted correctly. False positives (FP) are non-interacting proteins that are predicted as interacting proteins. False negatives (FN) are interacting proteins that are missed.

We did not perform cross validation to evaluate the SVM model. Instead, we prepared independent test sets that were not used in training the SVM model. In general, cross-validation shows a much higher performance than testing a prediction model on a new data set that was not used in training. As shown later in this paper, some virus proteins have a very small number of human protein interaction partners to perform cross validation. For example, the HCV NS4A and HCV NS4B proteins have only 7 and 1 interaction partners, respectively. The HPV E4 protein has only 2 interaction partners, and the HPV E8 protein has only 7 interaction partners. Thus, we tested the SVM model on new, independent data sets that were not used in training the model.

\section{Interaction of HCV proteins}

Due to the randomness in drawing negative data from HPRD and positive data from the data set of HCVhuman protein interactions for the training set, we prepared three independent test sets and evaluated the SVM model with the sets (Additional file 1). For comparison, we also tested the method of Shen et al. [5] on these sets. Table 3 compares our method with Shen's method in terms of sensitivity, specificity and accuracy with the $\mathrm{HCV}$ data.

As shown in Table 3, our SVM model, on average, achieved a sensitivity of $77.8 \%$, a specificity of $85.4 \%$ and an accuracy of $81.6 \%$. It outperformed the method of Shen $e t$ al. [5], which on average, achieved a sensitivity of $74.0 \%$, a specificity of $80.4 \%$ and an accuracy of $77.1 \%$. In particular, our method showed the best performance in the second test set.

To find new human proteins that potentially interact with $\mathrm{HCV}$ proteins $\left(\mathrm{H}_{\mathrm{HCV}}\right)$, we ran BLAST http://www. ncbi.nlm.nih.gov/BLAST/ with the known $\mathrm{H}_{\mathrm{HCV}}$ as query sequences against the human proteins in GenBank http://www.ncbi.nlm.nih.gov/genbank/. The E-value was set to $10^{-20}$ when running BLAST. After removing redundant sequences with the 695 known $\mathrm{H}_{\mathrm{HCV}}$ proteins, we obtained a total of 4,209 human proteins as the initial candidates of $\mathrm{H}_{\mathrm{HCV}}$ (Table 4).

In the 4,209 human proteins, the SVM model predicted 1,180 proteins as potential candidates of $\mathrm{H}_{\mathrm{HCV}}$. The 1,180 candidates of $\mathrm{H}_{\mathrm{HCV}}$ were refined further by selecting human proteins that have the same gene ontology (GO) cellular component terms [20] as the known $\mathrm{H}_{\mathrm{HCV}}$ for each HCV protein. After this refinement, we obtained a total of 456 candidates of $\mathrm{H}_{\mathrm{HCV}}$. For instance, the HCV E2 protein has 29 known $\mathrm{H}_{\mathrm{HCV}}$ proteins, and the $29 \mathrm{H}_{\mathrm{HCV}}$ proteins have a total of $15 \mathrm{GO}$ cellular component terms. The SVM model predicted $33 \mathrm{H}_{\mathrm{HCV}}$ proteins as interacting partners of the HCV E2 protein, and 10 out of the 33

Table 3 Comparison of two representation methods for predicting human proteins interacting with HCV proteins

\begin{tabular}{ccccccc}
\hline \multirow{2}{*}{ Test set } & \multicolumn{3}{c}{ Our representation } & \multicolumn{3}{c}{ Shen's representation } \\
\cline { 2 - 7 } & SN (\%) & SP (\%) & AC (\%) & SN (\%) & SP (\%) & AC (\%) \\
\hline 1 & 75.9 & 83.6 & 79.7 & 73.8 & 82.1 & 77.9 \\
$\mathbf{2}$ & $\mathbf{8 0 . 5}$ & $\mathbf{8 9 . 7}$ & $\mathbf{8 5 . 1}$ & $\mathbf{7 3 . 8}$ & $\mathbf{8 2 . 1}$ & $\mathbf{7 7 . 9}$ \\
3 & 76.9 & 83.1 & 80 & 74.4 & 76.9 & 75.6 \\
\hline Average & 77.8 & 85.4 & 81.6 & 74.0 & 80.4 & 77.1
\end{tabular}

Comparison of our representation method with the method by Shen et al. [5] in terms of sensitivity (SN), specificity (SP) and accuracy (AC) with the HCV data. The two representation methods are different in their classification of 20 amino acids and definition of the relative frequency of an amino acid triplet (see Methods section for details). 
Table 4 New human proteins found by our method as potential interaction partners with HCV proteins

\begin{tabular}{ccccc}
\hline $\begin{array}{c}\text { HCV } \\
\text { protein }\end{array}$ & $\begin{array}{c}\text { Known } \\
\mathbf{H}_{\text {HCV }}\end{array}$ & $\begin{array}{c}\text { Initial candidates of } \mathbf{H}_{\text {HCV }} \text { by BLAST } \\
\text { search }\end{array}$ & $\begin{array}{c}\text { Predicted candidates of } \mathbf{H}_{\mathbf{H C V}} \text { by } \\
\text { SVM }\end{array}$ & $\begin{array}{c}\text { Refined candidates of } \mathbf{H}_{\text {HCV }} \text { with } \\
\text { GO }\end{array}$ \\
\hline Core & 118 & 732 & 225 & 71 \\
E1 & 16 & 150 & 28 & 9 \\
E2 & 29 & 182 & 33 & 10 \\
F & 10 & 206 & 57 & 12 \\
NS2 & 11 & 176 & 42 & 8 \\
NS3 & 298 & 1,599 & 495 & 195 \\
NS4A & 7 & 114 & 33 & 10 \\
NS4B & 1 & 1 & 1 & 1 \\
NS5A & 152 & 499 & 123 & 72 \\
NS5B & 36 & 384 & 92 & 51 \\
p7 & 17 & 166 & 51 & 17 \\
\hline Total & 695 & 4,209 & 1,180 & 456 \\
\hline
\end{tabular}

The 'Initial candidates of $\mathrm{H}_{\mathrm{HCV}}$ by BLAST search' indicate the initial candidates of human proteins interacting with HCV proteins (called $\mathrm{H}_{\mathrm{HCV}}$ in this paper) found by BLAST search from GenBank with the known $\mathrm{H}_{\mathrm{HCV}}$ as query sequences. The 'Predicted candidates of $\mathrm{H}_{\mathrm{HCV}}$ by SVM' were determined by the SVM model from the initial candidates of $\mathrm{H}_{\mathrm{HCV}}$. The 'Refined candidates of $\mathrm{H}_{\mathrm{HCV}}$ with $\mathrm{GO}^{\prime}$ were obtained from the predicted candidates by selecting $\mathrm{H}_{\mathrm{HCV}}$ that have the same $\mathrm{GO}$ cellular component terms as the known $\mathrm{H}_{\mathrm{HCV}}$.

candidates were left as reliable candidates of $\mathrm{H}_{\mathrm{HCV}}$, since they have the same GO cellular component terms as the known $\mathrm{H}_{\mathrm{HCV}}$ proteins. Figure 1 shows an interaction network of the $456 \mathrm{H}_{\mathrm{HCV}}$ proteins predicted by our method.

\section{Interaction of HPV proteins}

To evaluate the performance of the model with the HPV datasets, we prepared three training sets and three test sets (Additional file 2). We tested both our method and Shen's

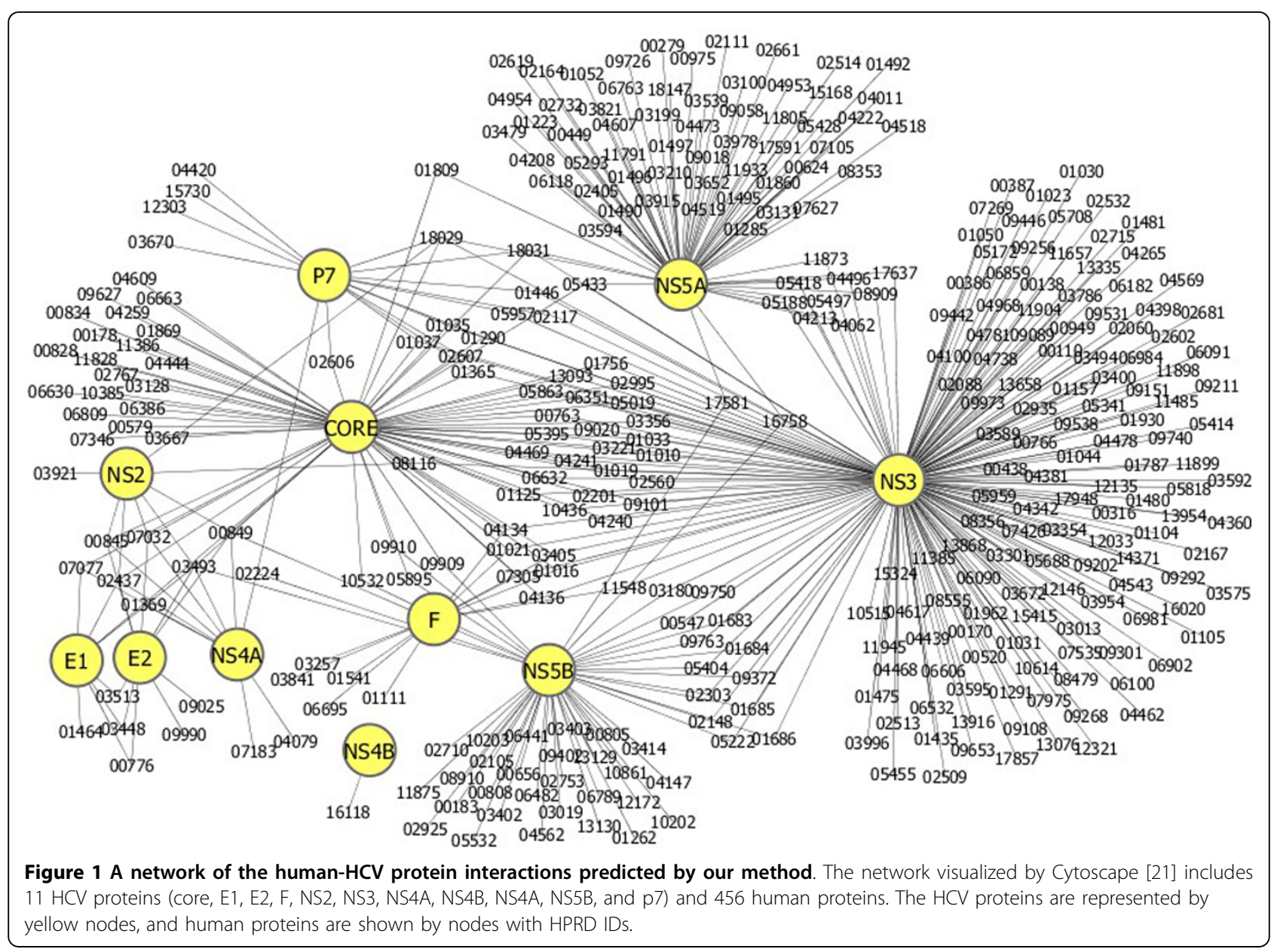


method [5] on the test sets. As shown in Table 5, our method achieved on average, a sensitivity of $78.8 \%$, a specificity of $87.8 \%$ and an accuracy of $83.3 \%$. Shen's method showed, on average, a sensitivity of $72.4 \%$, a specificity of $83.9 \%$ and an accuracy of $78.2 \%$. In both HCV and HPV data sets, our method was better than Shen's method. The major difference between our method and Shen's method is in the representation of protein sequences. Our classification of 20 amino and definition of the relative frequency of an amino acid triplet are different from those of Shen's method (see the Methods section for details).

To find new human proteins that potentially interact with HPV proteins $\left(\mathrm{H}_{\mathrm{HPV}}\right)$, we ran BLASTP http://www. ncbi.nlm.nih.gov/BLAST/ with the E-value $\leq 10^{-20}$ against the human proteins in GenBank http://www.ncbi. nlm.nih.gov/genbank/. After removing redundant sequences with the 252 known $\mathrm{H}_{\mathrm{HPV}}$ proteins, we obtained a total of 560 human proteins as the initial candidates of $\mathrm{H}_{\mathrm{HPV}}$ (Table 6). In the $560 \mathrm{H}_{\mathrm{HPV}}$ proteins, the SVM model predicted 156 proteins as potential candidates of $\mathrm{H}_{\mathrm{HPV}}$. In the $156 \mathrm{H}_{\mathrm{HCV}}$ proteins, we selected the human proteins that have the same cellular component GO IDs as the known $\mathrm{H}_{\mathrm{HPV}}$ for each HPV protein. As a result, we found a total of 130 human proteins as potential $\mathrm{H}_{\mathrm{HPV}}$. Figure 2 shows an interaction network of 130 $\mathrm{H}_{\mathrm{HPV}}$ proteins that were predicted by our method.

\section{Comparative analysis of two viral interaction networks}

In viral infections, different viruses target different human proteins, so they usually have interaction partners. We compared the interaction network of HCV with that of HPV to find common human protein targets. Figure $3 \mathrm{~A}$ shows the $\mathrm{HCV}$ interaction network overlaid by the HPV interaction network, both for the known interaction data. HCV and HPV have 11 human proteins as their common interaction partners: STAT3 (HPRD 00026), CDKN1A (HPRD 00298), NR4A1 (HPRD 00744), JUN (HPRD 01302), TP53 (HPRD 01859), TP73 (HPRD 03587), IPO5 (HPRD 03597), FADD (HPRD 03909), FHL2 (HPRD 04026), EP300 (HPRD 04078), and AHNAK (HPRD 14684). In particular, four human proteins, CDKN1A (HPRD 00298), TP53 (HPRD 01859), TP73 (HPRD 03587), and FADD (HPRD 03909) proteins, shown in Figure 3B, are the common interaction partners of the HCV core protein and HPV E6 protein. They are known to be engaged in the p53 signalling pathway for apoptosis http://www.sabiosciences.com/rt_pcr_product/ HTML/PAHS-027A.html.

In a similar way, we compared the predicted interaction network of HCV and that of HPV (Figure 4). HCV and HPV have 7 human proteins as their common interaction partners (pink nodes in the network): SLC1A1 (HPRD 00597), KRT17 (HPRD 01019), TP63 (HPRD 04469), GSK3B (HPRD 05418), CDK19 (HPRD 07627), CDK11B (HPRD 08909), and NLK (HPRD 17637).

\section{Conclusions}

Most methods for predicting protein-protein interactions focus on the interactions within a species rather than for the interactions across different species, such as interactions between virus and host cell proteins. In this paper we presented a support vector machine (SVM) model and its representation method for predicting the interactions between viruses and human proteins.

We represented a protein sequence using three consecutive amino acids called amino acid triplet. We mapped a protein sequence of variable length to a feature vector of fixed length, and then mapped the feature vector to a frequency vector that represents the relative frequency of each triplet within the protein sequence. The SVM model showed an average accuracy of $81.6 \%$ in predicting human proteins that interact with $\mathrm{HCV}$ proteins, and a similar accuracy $(83.3 \%)$ in predicting human proteins that interact with HPV proteins. The performance of our SVM model was better than that of the other method [5] in both data sets of HCV and HPV. Using the SVM model and Gene Ontology (GO) annotations of proteins, we also predicted new human proteins that potentially interact with HCV or HPV proteins. From the comparative analysis of two viral interaction networks, we found common human proteins that are targeted by both viruses.

Our experiment on 2 different types of viruses showed that encoding the relative frequency of amino acid triplets of a protein sequence is a simple yet powerful representation method for protein sequences when

Table 5 Comparison of two methods for predicting human proteins interacting with HPV proteins

\begin{tabular}{ccccccc}
\hline Test set & \multicolumn{3}{c}{ Our representation } & \multicolumn{3}{c}{ Shen's representation } \\
\cline { 2 - 7 } & SN (\%) & SP (\%) & AC (\%) & SN (\%) & SP (\%) & AC (\%) \\
\hline $\mathbf{1}$ & $\mathbf{8 6 . 5}$ & $\mathbf{8 8 . 5}$ & $\mathbf{8 7 . 5}$ & $\mathbf{6 9 . 2}$ & $\mathbf{8 4 . 6}$ & 76.9 \\
2 & 73.1 & 88.5 & 80.8 & 69.2 & 84.6 & 82.9 \\
3 & 76.9 & 86.5 & 81.7 & 78.8 & 80.8 & 78.2 \\
\hline Average & 78.8 & 87.8 & 83.3 & 72.4 & 83.9 & 78.2 \\
\hline
\end{tabular}

Comparison of our representation method with the method by Shen et al. [5] in terms of sensitivity (SN), specificity (SP) and accuracy (AC) with the HPV data. 
Table 6 New human proteins found by our method as potential interaction partners with HPV proteins

\begin{tabular}{ccccc}
\hline $\begin{array}{c}\text { HPV } \\
\text { protein }\end{array}$ & $\begin{array}{c}\text { Known } \\
\mathbf{H}_{\text {HPV }}\end{array}$ & $\begin{array}{c}\text { Initial candidates of } \mathbf{H}_{\text {HPV }} \text { by BLAST } \\
\text { search }\end{array}$ & $\begin{array}{c}\text { Predicted candidates of } \mathbf{H}_{\mathbf{H P V}} \text { by } \\
\text { SVM }\end{array}$ & $\begin{array}{c}\text { Refined candidates of } \mathbf{H}_{\text {HPV }} \text { with } \\
\mathbf{G O}\end{array}$ \\
\hline E1 & 9 & 90 & 7 & 6 \\
E2 & 36 & 157 & 28 & 21 \\
E4 & 2 & 11 & 2 & 2 \\
E5 & 13 & 34 & 25 & 23 \\
E6 & 78 & 103 & 38 & 29 \\
E7 & 76 & 100 & 35 & 29 \\
E8 & 7 & 19 & 8 & 8 \\
L1 & 20 & 39 & 8 & 7 \\
L2 & 11 & 7 & 5 & 5 \\
\hline Total & 252 & 560 & 156 & 130 \\
\hline
\end{tabular}

The 'Initial candidates of $\mathrm{H}_{\mathrm{HPV}}$ by BLAST search' indicate the initial candidates of human proteins interacting with HPV proteins ( $\mathrm{H}_{\mathrm{HPV}}$ ) found by BLAST search from GenBank with the known $\mathrm{H}_{\mathrm{HCV}}$ as query sequences. The 'Predicted candidates of $\mathrm{H}_{\mathrm{HPV}}$ by SVM' were determined by the SVM model from the initial candidates of $\mathrm{H}_{\mathrm{HPV}}$. The 'Refined candidates of $\mathrm{H}_{\mathrm{HPV}}$ with $\mathrm{GO}$ ' were obtained from the predicted candidates by selecting $\mathrm{H}_{\mathrm{HCV}}$ that has the same $\mathrm{GO}$ cellular component terms as the known $\mathrm{H}_{\mathrm{HPV}}$.

finding protein-protein interactions across different species. The representation method has several advantages. First, it enables a prediction model to achieve better performance than that of other representations. Second, it generates feature vectors of fixed length regardless of the sequence length. Third, the same representation is applicable to different types of proteins.
Elucidating virus-host interactions is important for understanding viral infections and for identifying new targets for rational drug discovery. In the future, we plan to construct virus-host protein interaction networks to achieve more viral species and perform further comparative analysis of the interaction networks to discover interaction patterns central or specific to them.

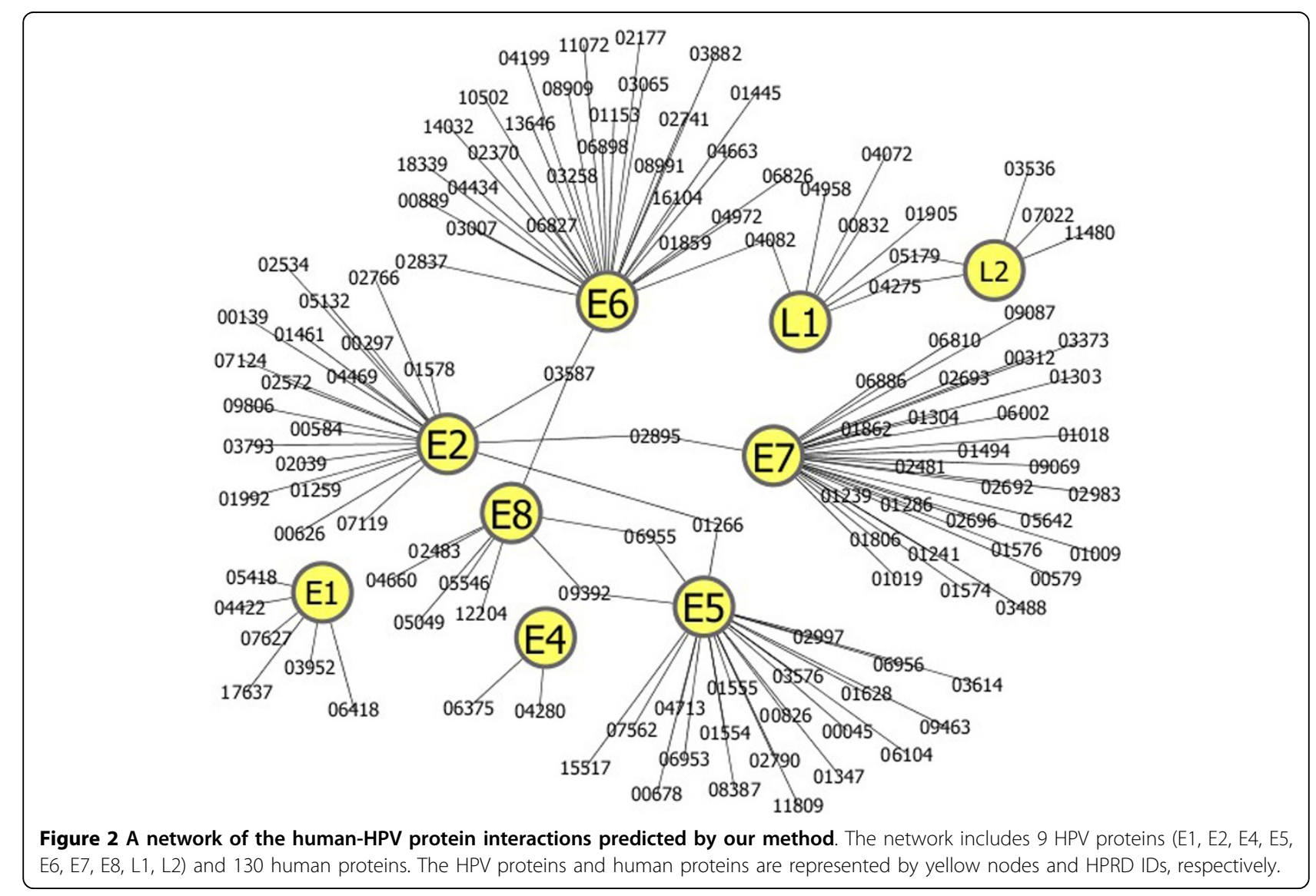




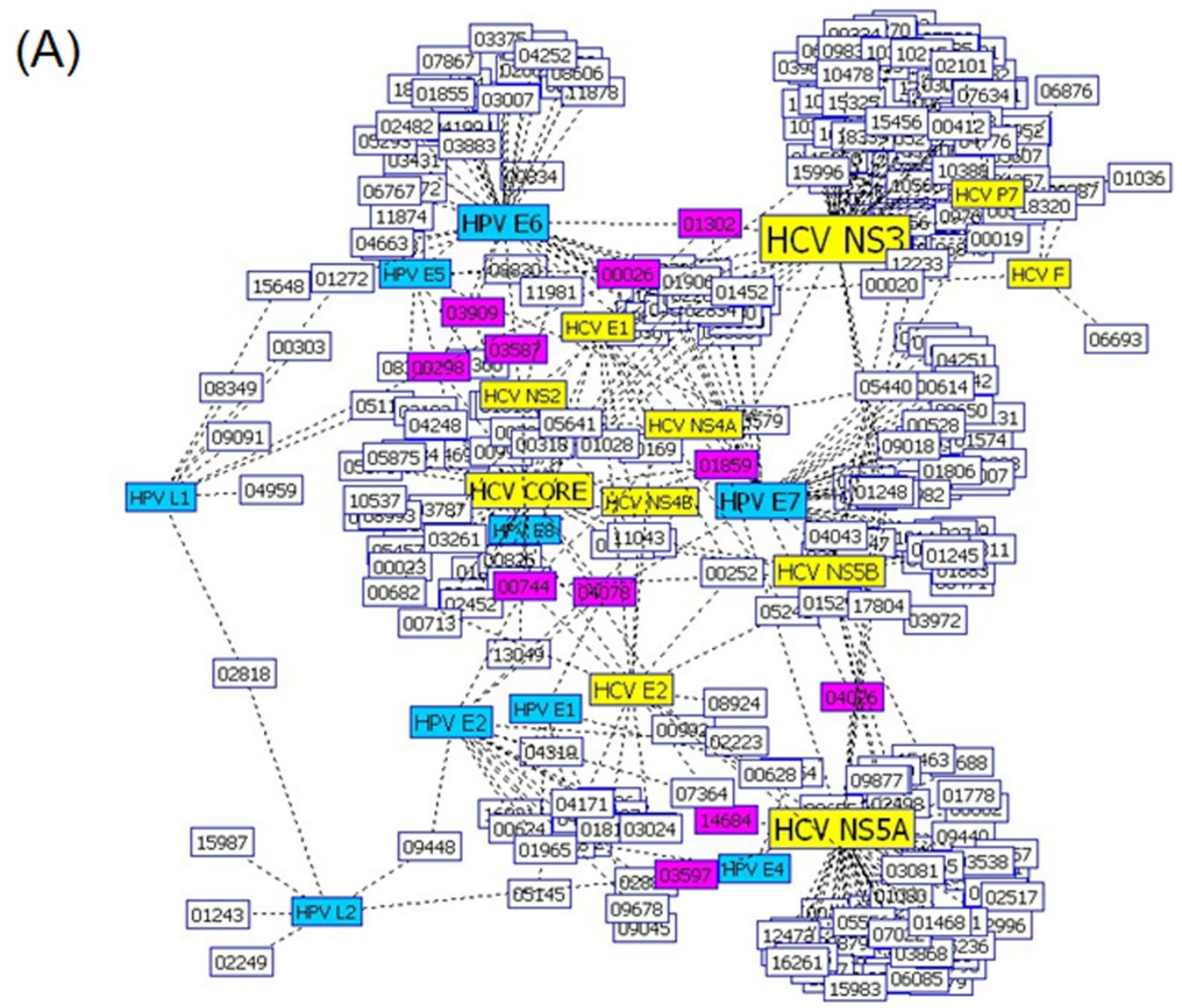

(B)

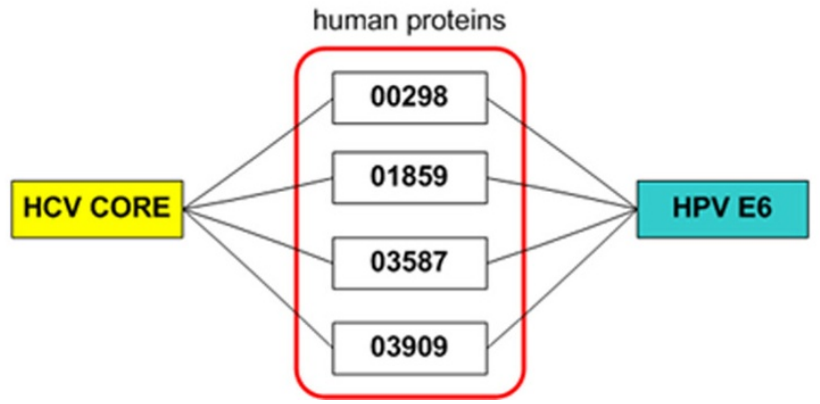

Figure 3 Comparison of the HCV interaction network with the HPV interaction network for the known interactions. (A) The HCV interaction network is overlaid by the HPV interaction network by WeblnterViewer [22]. Both networks show the known interactions of HCV and HPV with human proteins. HCV and HPV have 11 human proteins as their common interaction partners: STAT3 (HPRD 00026), CDKN1A (HPRD 00298), NR4A1 (HPRD 00744), JUN (HPRD 01302), TP53 (HPRD 01859), TP73 (HPRD 03587), IPO5 (HPRD 03597), FADD (HPRD 03909), FHL2 (HPRD 04026), EP300 (HPRD 04078), and AHNAK (HPRD 14684). Pink node: human protein interacting with both HCV and HPV proteins; yellow node: HCV protein, cyan node: HPV protein; white node: human protein interacting with either HCV protein or HPV protein but not both. (B) The CDKN1A (HPRD 00298), TP53 (HPRD 01859), TP73 (HPRD 03587), and FADD (HPRD 03909) proteins in the red box are the common interaction partners of the HCV core protein and HPV E6 protein. They are known to be engaged in the p53 signaling pathway for apoptosis http://www. sabiosciences.com/rt_pcr_product/HTML/PAHS-027A.html. 


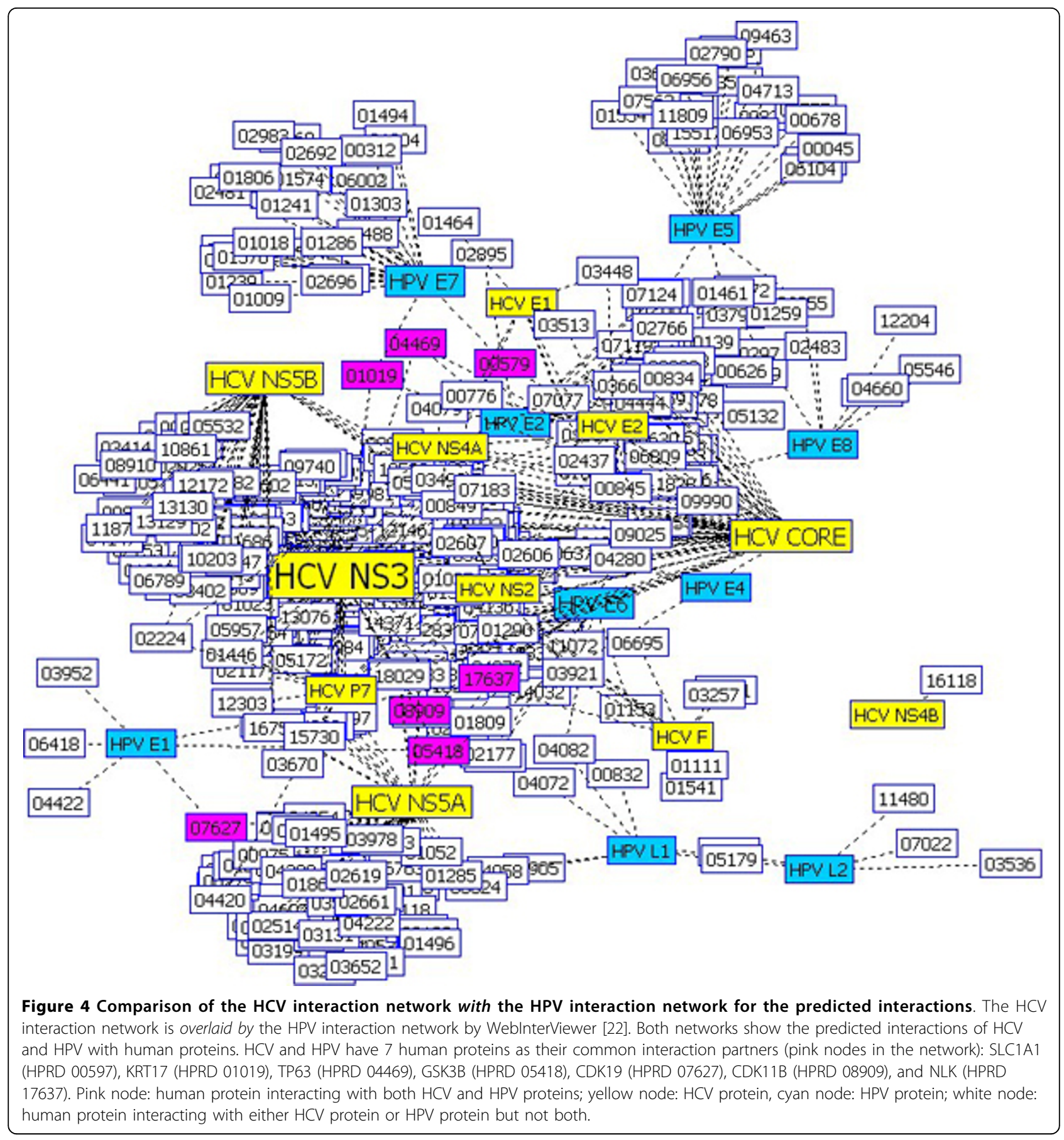

\section{Additional material}

Additional file 1: Three data sets used for predicting human proteins interacting with HCV proteins. Three training and test sets of human proteins (HPRD IDs) that were used for the results of Table 3. Additional file 2: Three data sets used for predicting human proteins interacting with HPV proteins. Three training and test sets of human proteins (HPRD IDs) that were used for the results of Table 5.

\section{Acknowledgements}

This research was supported by Basic Science Research Program through the National Research Foundation of Korea (NRF) funded by the Ministry of Education, Science and Technology (2011-0003766).

This article has been published as part of BMC Bioinformatics Volume 13 Supplement 7, 2012: Advanced intelligent computing theories and their applications in bioinformatics. Proceedings of the 2011 International Conference on Intelligent Computing (ICIC 2011). The full contents of the supplement are available online at http://www.biomedcentral.com/ bmcbioinformatics/supplements/13/S7. 


\section{Authors' contributions}

Chao Fang did the early work with HCV data, and Guangyu Cui finished the work with the HPV data and prepared the first draft of the manuscript. Kyungsook Han supervised the work and rewrote the manuscript.

\section{Competing interests}

The authors declare that they have no competing interests.

Published: 8 May 2012

\section{References}

1. Bock JR, Gough DA: Predicting protein-protein interactions from primary structure. Bioinformatics 2001, 17:455-460.

2. Martin S, Roe D, Faulon JL: Predicting protein-protein interactions using signature products. Bioinformatics 2005, 21:218-226.

3. Xia JF, Zhao XM, Huang DS: Predicting protein-protein interactions from protein sequences using meta predictor. Amino Acids 2010, 39:1595-1599.

4. Guo Y, Yu L, Wen Z, Li M: Using support vector machine combined with auto covariance to predict protein-protein interactions from protein sequences. Nucleic Acids Res 2008, 36:3025-3030.

5. Shen J, Zhang J, Luo X, Zhu W, Yu K, Li Y, Jiang H: Predicting proteinprotein interactions based only on sequences information. Proc Natl Acad Sci USA 2007, 104:4337-4341.

6. Shi MG, Xia JF, Li XL, Huang DS: Predicting protein-protein interactions from sequence using correlation coefficient and high-quality interaction dataset. Amino Acids 2010, 38:891-899.

7. Wu XM, Zhu L, Guo J, Zhang DY, Lin K: Prediction of yeast protein-protein interaction network: insights from the Gene Ontology and annotations. Nucleic Acids Res 2006, 34:2137-2150.

8. Nanni L: Fusion of classifiers for predicting protein-protein interactions. Neurocomputing 2005, 68:289-296

9. You ZH, Lei YK, Huang DS, Zhou XB: Using manifold embedding for assessing and predicting protein interactions from high-throughput experimental data. Bioinformatics 2010, 26:2744-2751.

10. Gomez SM, Noble WS, Rzhetsky A: Learning to predict protein-protein interactions from protein sequences. Bioinformatics 2003, 19:1875-1881.

11. Taylor WR: The classification of amino acid conservation. J Theor Biol 1986, 119:205-218

12. Leslie CS, Eskin E, Cohen A, Weston J, Noble WS: Mismatch string kernels for discriminative protein classification. Bioinformatics 2004, 20:467-476.

13. Furey TS, Cristianini N, Duffy N, Bednarski DW, Schummer M, Haussler D: Support vector machine classification and validation of cancer tissue samples using microarray expression data. Bioinformatics 2000, 16:906-914.

14. Joachims T: Making large scale SVM learning practical. In Advances in Kernel Methods-Support Vector Learning. Cambridge: MIT Press;Scholkopf, Burges, Smola 1999:

15. Kato N: Genome of human hepatitis C virus (HCV): gene organization, sequence diversity, and variation. Microb Comp Genomics 2000, 5:129-151.

16. Appel N, Schaller T, Penin F, Bartenschlager R: From structure to function: New insights into hepatitis C virus RNA replication. J Biol Chem 2006, 281:9833-9836.

17. de Chassey B, Navratil V, Tafforeau L, Hiet MS, Aublin-Gex A, Agaugué S, Meiffren G, Pradezynski F, Faria BF, Chantier T, Le Breton M, Pellet J, Davoust N, Mangeot PE, Chaboud A, Penin F, Jacob Y, Vidalain PO, Vidal M, André $P$, Rabourdin-Combe $C$, Lotteau V: Hepatitis $C$ virus infection protein network. Mol Syst Biol 2008, 4:230.

18. Lowy DR, Schiller JT: Prophylactic human papillomavirus vaccines. J Clin Invest 2006, 116:1167-1173.

19. Chaturvedi A, Gillson ML: Human papillomavirus and head and neck cancer. In Epidemiology, Pathogenesis, and Prevention of Head and Neck Cancer.. 1 edition. New York: Springer;Olshan AF 2010:

20. Ashburner M, Ball CA, Blake JA, Botstein D, Butler H, Cherry JM, Davis AP, Dolinski K, Dwight SS, Eppig JT, Harris MA, Hill DP, Issel-Tarver L, Kasarskis A, Lewis S, Matese JC, Richardson JE, Ringwald M, Rubin GM, Sherlock G: Gene ontology: tool for the unification of biology. Nat Genet 2000, 25:25-29.

21. Shannon P, Markiel A, Ozier O, Baliga NS, Wang JT, Ramage D, Amin N, Schwikowski B, Ideker T: Cytoscape: a software environment for integrated models of biomolecular interaction networks. Genome Res 2003, 13:2498-2504.
22. Han $\mathrm{K}$, Ju B, Jung $\mathrm{H}$ : WeblnterViewer: integrated framework for visualizing and analyzing molecular interaction networks. Nucleic Acids Res 2004, 32:W89-W95.

doi:10.1186/1471-2105-13-S7-S5

Cite this article as: Cui et al:: Prediction of protein-protein interactions between viruses and human by an SVM model. BMC Bioinformatics 2012 13(Suppl 7):S5

\section{Submit your next manuscript to BioMed Central and take full advantage of:}

- Convenient online submission

- Thorough peer review

- No space constraints or color figure charges

- Immediate publication on acceptance

- Inclusion in PubMed, CAS, Scopus and Google Scholar

- Research which is freely available for redistribution 